\title{
Primary Retroperitoneal Mucinous Tumor; Report of Two Cases and Review of Literature
}

\author{
Ali Dastranj Tabrizi 1 ${ }^{*}$, Amir Vahedi ${ }^{2}$, Manizheh Sayyah-Melli ${ }^{3}$, Mehri Jafari Shobeiri ${ }^{3}$, \\ Parvin Mostafa Gharabaghi ${ }^{3}$, Elaheh Ouladsahebmadarek ${ }^{1}$
}

\section{Article History:}

Received 9 October 2013

Accepted 7 January 2014

Revised 23 December 2013

Available online 7 January 2014

\section{Keywords: \\ Cystadenoma \\ Mucinous Adenocarcinoma \\ Retroperitoneal Space}

\section{Corresponding Author:}

Ali Dastranj Tabrizi, Associate Professor of Pathology, Women's Reproductive Health Research Center, Tabriz University of Medical Sciences, Tabriz, Iran. Tel: +989144069887

Email: dastranjt@gmail.com

\begin{abstract}
Primary retroperitoneal mucinous tumors are rare neoplasms and almost exclusively seen in women. In this case report we present two cases of this entity with their clinicopathologic features. The first patient was a 32 years old unmarried woman presented with abdominal pain. Ultrasound imaging demonstrated a solid and thick walled ovarian mass $\mathrm{m}$. 105* $70^{*} 80 \mathrm{~mm}$. At laparotomy a large retroperitoneal mass with extention to pelvis, dome of urinary bladder and upper abdomen was found and omentum was involved by the tumor. Histopathological examination revealed a mucinous adenocarcinoma with mural nodule contained high-grade anaplastic carcinoma in retroperitoneal space. The second case was a 36 years old woman with retroperitoneal mucinous cystadenoma. The first case died of disease at first month of her diagnosis. Primary retroperitoneal mucinous tumors that containing anaplastic carcinoma or sarcomas are highly aggressive tumors with poor prognosis showing metastasis by high-grade component.
\end{abstract}

1- Associate Professor of Pathology, Women's Reproductive Health Research Center, Tabriz University of Medical Sciences, Tabriz, Iran.

2- Associate Professor of Pathology, Department of Pathology, Tabriz University of Medical Sciences, Tabriz, Iran.

3- Full Professor of Ob \& Gyn, Women's Reproductive Health Research Center, Tabriz University of Medical Sciences, Tabriz, Iran. 


\section{Introduction:}

Primary retroperitoneal mucinous tumors (PRMTs) are rare neoplasm and almost exclusively seen in women $(1,2,3)$. Recently the largest case series composed of 18 cases has been published (4). Similar to the ovarian mucinous tumors, PRMTs composed of benign, borderline and malignant tumors. In this case report we present two patients with retroperitoneal mucinous cystadenoma and cystadenocarcinoma with emphasize on poor outcome of patients with anaplastic component and discuss on immunohistochemical profile of this undifferentiated malignant tumor.

\section{Case Report}

Case 1:

A 32 years old unmarried lady admitted with recurrent abdominal pain in RLQ for one year, accentuation of her symptoms in a week and abdominal distention in the last three days. Her physical examination revealed an abdominal mass in the RLQ in association with - tenderness and rebound tenderness. Her clinical history was unremarkable. Ultrasound imaging demonstrated a solid and thick walled ovarian mass $\mathrm{m}$. 105*70*80 mm. The uterus was normal in size. Serum CA-125 was 40 $\mathrm{ng} / \mathrm{ml}$ (normal 0-35ng/ml). At laparotomy a large retroperitoneal mass with extention to pelvis, dome of urinary bladder and upper abdomen was found. Omentum was involved by the tumor. In pelvic exploration uterus and ovaries were normal in size and appearance. Total resection of the mass in association with partial omentectomy was performed and the specimens sent for histopathological examination. The patient was expired after two weeks of operation.

The received specimen consisted of multiple fragments of creamy-brown colored solid and membranous fragments with soft consistency m. up to $10^{*} 6^{*} 2 \mathrm{~cm}$. The larger fragment contained firm foci with protrusion to the cyst lumen in association with hemorrhage. Microscopically the cyst lining showed an abnormal cellular proliferation composed of mucinous cells in tall and thin papillary fronds. The papillaes composed of fibrovascular cores and lined by malignant appearing epithelial cells consistent with mucinous intraepithelial carcinoma (fig 1A). Other sections demonstrated infiltrative invasive foci in association with undifferentiated malignant cellular growth in the solid mural nodule (fig 1B). Sections of the received omentum showed metastatic foci by the anaplastic component (fig 1C). No ectopic ovarian tissue and no residual dermoid cyst was found in the microscopic sections, therefore a diagnosis of a primary retroperitoneal mucinous carcinoma(infiltrative type) with anaplastic carcinoma or pleomorphic sarcoma in the mural nodule was made.

Immunostaining performed on representative sections for CK7, CK20, EMA, Desmin, Myogenin, S100 and Vimentin. The different foci in the tumor including intraepithelial, well differentiated invasive and anaplastic regions showed diffuse positive reaction for CK 7 (fig. 1D). Reaction for EMA was positive in intraepithelial and well differentiated foci but negative in anapalstic areas. Vimentin showed positivity only in anaplastic foci of the malignant case (Fig. 1E). Diffuse staining for S100 protein was found in all foci of the malignant case. Staining for CK20, Desmine, Myogenin were completely negative in all foci of the malignant case.

\section{Case 2 :}

A 36 years old lady was referred to our center with abdominal pain for 8 years. Her chief complaint was accentuated in the last two months in association with abnormal vaginal bleeding. In physical examination there was a firm and mobile mass in the right side of the abdomen with no tenderness. In laparotomy, uterus, ovaries, intestine and omentum were normal but a retroperitoneal cyst $\mathrm{m} .10 * 5 \mathrm{~cm}$ with no adhesion was noted and easily dissected. The resected specimen sent for histologic evaluation. In macroscopic examination the specimen consisted of cystic structure m. $4 * 4 * 4 \mathrm{~cm}$ with no solid focus .In cut sections the inner and outer surfaces of the cyst were smooth and the wall thickness was $2 \mathrm{~mm}$. In microscopic examination, the cyst was lined by single layer of mucinous epithelium with no stratification, no nuclear atypia and no mitotic figure (Fig2A) . Additional microscopic sections were taken for rule out of borderline lesion and no focus of 
abnormal proliferation was noted. Therefore, diagnosis of retroperitoneal mucinous cystadenoma was confirmed. Immunohistochemically the mucinous lining was positive for CK7 and negative for CK20 (Fig2B).

\section{Discussion:}

The age and clinical presentation of both of our cases were according to literature $(3,4)$. However in contrast to literature both of these cases presented with pain in addition to abdominal mass. The preoperative diagnosis in our cases was ovarian cyst. Since the symptoms are nonspecific and because there is no sensitive detecting method or reliable marker, usually the diagnosis is difficult prior to surgery $(5,6)$.

Our first case died of disease at first month of her diagnosis due to highly aggressive clinical course. This rapid progressive and fatal outcome caused by massive abdominal and pelvic extension of tumor and omentum metastasis. The metastatic foci in the omentum consisted exclusively of high grade component (fig 1F). This fatal outcome is in line with other case reports of primary retroperitoneal mucinous cystadeno carcinomas with sarcomatous or carcinomatous mural nodule whereas only 2 of 17 retroperitoneal mucinous carcinoma without malignant mural nodule in the literature had aggressive clinical course (7). Primary retroperitoneal mucinous adenocarcinoma is a rare neoplasm and even rarer occurrence is mucinous cystadeno carcinoma with sarcomatous or anaplastic carcinomatous mural nodule. A mural nodule may composed of anaplastic carcinoma, sarcoma like tissue, a true sarcoma , carcinosarcoma or sarcomatoid carcinoma. Sarcoma like mural nodules in ovarian mucinous tumors were first described by Scully and Prat in $1977(8,9)$. Differentiation between anaplastic carcinoma and sarcoma in a mural nodule may be made by routine immuno histochemical markers (10), however, sarcoma like nodule may be seen in anaplastic carcinoma (11). Since bizarre spindle shaped nuclei and brisk mitotic count may be seen in both sarcoma like tissue and true sarcoma in a mural nodule , differentiation between two condition is more problematic. However, presence of monotonous cell population, poorly circumscription and large size favors diagnosis of sarcoma and vice versa. In our first case, diffuse staining for CK7 in all of malignant foci, positive EMA reaction in intracystic and well differentiated invasive foci, diffuse negativity for Desmin and myogenin in the anaplastic and undifferentiated regions of the mural nodule favors the diagnosis of anaplastic carcinoma in the mural nodule. Diffuse expression of S100-Protein and Vimentin in the undifferentiated foci is nonspecific as S-100 protein positivity may be seen in epithelial tumors and vimentin expression may be found in sarcomatoid carcinoma $(12,13)$.

For explanation of origin of these lesions, four main hypotheses have been proposed. These lesions may originate from ectopic ovarian tissue, monodermal teratoma, intestinal duplication or invagination of peritoneal mesothelial layer. Absence of ovarian stroma, intestinal wall and components of dermiod cyst is in contrast with the first three hypothesis. In the other hand positivity for CK7 and absence of CK20 in both cases is similar to expression pattern of these markers in ovary. These IHC findings support the forth hypothesis which suggests that these tumors arise from invagination of peritoneal mesothelial layer which undergoes mucinous metaplasia for cyst formation and tumor development. Since retroperitoneal endometriosis is a well-recognized condition, and rare cases of carcinosarcomas have been reported in retroperitoneum in association with endometriosis it may be reasonable to search for foci of endometriosis in cases of retroperitoneal mucinous tumors (14-17).

Since the largest case series of the retroperitoneal mucinous tumors consist only 18 cases and very rare occurrence of this entitiy, our review was limited by few numbers of cases.

In summary, we report two cases of primary retroperitoneal mucinous tumors. One of the cases had a rapidly fatal outcome due to metastatic spread of undifferentiated component, which found in the mural nodule. 


\section{Conflicts of interest:}

The authors declare no conflict of interest in this study
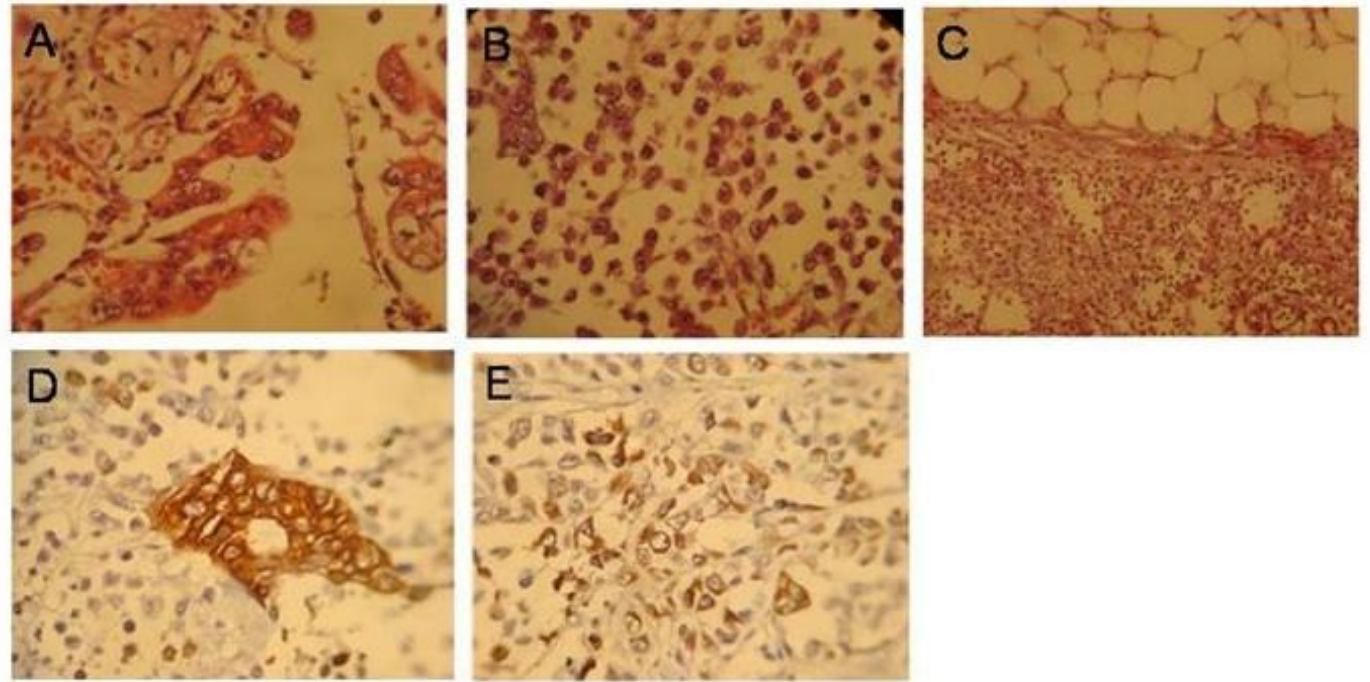

Figure 1. A. Mucinous borderline tumor with intraepithelial carcinoma (H\&E stain, X 400). B. Undifferentiated focus within mural nodule(H\&E stain,X400). C. Omentum with a metastatic focus by undifferentiated component(H\&E stain, XIOO). D. Positive CK 7 staining in infiltrative and destructive invasive focus (IHC, X400). E. Diffuse positive staining for Vimentin in undifferentiated malignant focus(IHC,400).
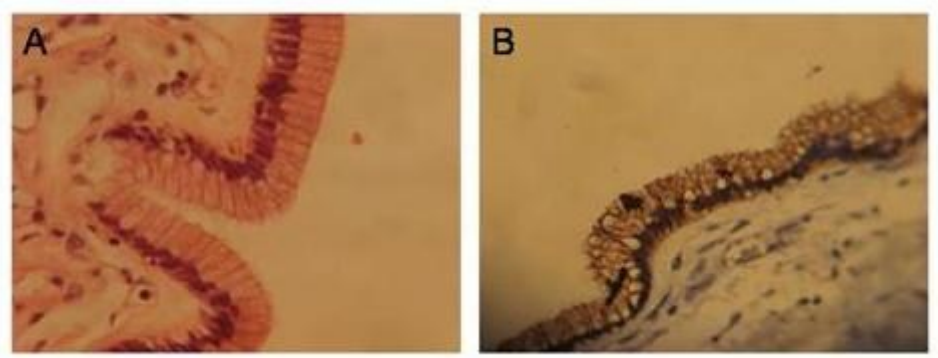

Figure 2 A, Benign mucinous lining of the cyst adenoma (H\&E,X400). B, Positive reaction for CK7 (IIIC,X400). 


\section{References:}

1. Thamboo TP, Sim R, Tan SY, Yap WM. Primary retroperitoneal mucinous ystadenocarcinoma in a male patient. J Clin Pathol. 2006; 59(6):655-7.

2. Motoyama T, Chida T, Fujiwara $T$, Watanabe $H$. Mucinous cystic tumor of the retroperitoneum. A report of two cases. Acta Cytol. 1994; 38(2):261-6.

3. Green JM, Bruner BC, Tang WW, Orihuela E. Retroperitoneal mucinous cystadenocarcinoma in a man: case report and review of the literature. Urol Oncol. 2007; 25(1):53-5.

4. Roma AA, Malpica A. Primary retroperitoneal mucinous tumors: a clinicopathologic study of 18 cases. Am J Surg Pathol. 2009; 33(4):526-33.

5. Isse K, Harada K, Suzuki Y, Ishiguro K, Sasaki M, Kajiura S, et al. Retroperitoneal mucinous cystadenoma: report of two cases and review of the literature. Pathol Int. 2004; 54(2):132-8.

6. Tangjitgamol S, Manusirivithaya S, Sheanakul C, Leelahakorn S, Thawaramara T, Kaewpila $\mathrm{N}$. Retroperitoneal mucinous cystadenocarcinoma: a case report and review of literature. Int $\mathrm{J}$ Gynecol Cancer. 2002; 12(4):403-8.

7. Mikami M, Tei C, Takehara K, Komiyama S, Suzuki A, Hirose T. Retroperitoneal primary mucinous adenocarcinoma with a mural nodule of anaplastic tumor: a case report and literature review. 2003; 22(2):205-8.

8. Prat J, Scully RE. Sarcomas in ovarian mucinous tumors: a report of two cases. Cancer. 1979; 44(4):1327-31.

9. Prat J, Scully RE. Ovarian mucinous tumors with sarcoma-like mural nodules: a report of seven cases. Cancer. 1979; 44(4):1332-44.

10. Fan YS, Thomas TM, Ip PP, Cheung AN. Osteoid-forming sarcoma-like mural nodule in a retroperitoneal mucinous cystadenocarcinoma. Histopathology. 2006; 49(2):201-4.

11. Fujii S, Konishi I, Kobayashi F, Okamura H, Yamabe H, Mori T. Sarcoma-like mural nodules combined with a microfocus of anaplastic carcinoma in mucinous ovarian tumor. Gynecol Oncol. 1985; 20(2):219-33.

12. Hu L, Lau SH, Tzang CH, Wen JM, Wang W, Xie D, Huang M, Wang Y, Wu MC, Huang JF, Zeng WF, Sham JS, Yang M, Guan XY. Association of Vimentin overexpression and hepatocellular carcinoma metastasis. Oncogene. 2004 8; 23(1):298-302.

13. Sekido Y, Satoh F, Usui Y, Tsutsumi Y. Sarcomatoid carcinoma of the renal pelvis: a case report. Pathol Int. 2000; 50(7):562-7.

14. Susini T, Massi D, Massi GB. Ureteral obstruction due to retroperitoneal endometriosis: a conservative approach including surgery and GnRH analogs. 1996; 10(2):129-31. 
15. Kelemen PR. Extrapelvic retroperitoneal endometrioma. Am J Surg. 2002; 184(1):52-3.

16. Rana S, Stanhope RC, Gaffey T, Morrey BF, Dumesic DA. Retroperitoneal endometriosis causing unilateral hip pain. Obstet Gynecol. 2001; 98(5 Pt 2):970-2.

17. Booth C, Zahn CM, McBroom J, Maxwell GL. Retroperitoneal müllerian carcinosarcoma associated with endometriosis: a case report. Gynecol Oncol. 2004; 93(2):546-9. 